\title{
TWO-SIDED BOUNDS FOR THE VOLUME OF RIGHT-ANGLED HYPERBOLIC POLYHEDRA
}

\author{
DUŠAN REPOVŠ AND ANDREI VESNIN
}

\begin{abstract}
For a compact right-angled polyhedron $R$ in $\mathbb{H}^{3}$ denote by $\operatorname{vol}(R)$ the volume and by $\operatorname{vert}(R)$ the number of vertices. Upper and lower bounds for $\operatorname{vol}(R)$ in terms of $\operatorname{vert}(R)$ were obtained in $[3$. Constructing a 2-parameter family of polyhedra, we show that the asymptotic upper bound $5 v_{3} / 8$, where $v_{3}$ is the volume of the ideal regular tetrahedron in $\mathbb{H}^{3}$, is a double limit point for ratios $\operatorname{vol}(R) / \operatorname{vert}(R)$. Moreover, we improve the lower bound in the case $\operatorname{vert}(R) \leqslant 56$.
\end{abstract}

\section{Right-ANGLED POLYHEDRA IN $\mathbb{H}^{3}$.}

In any space, right-angled polyhedra are very convenient to serve as "building blocks" for various geometric constructions. In particular, they have several interesting properties in hyperbolic 3 -space $\mathbb{H}^{3}$. One can try to obtain a hyperbolic 3-manifold using a right-angled polyhedron as its fundamental polyhedron. Or, one can construct a hyperbolic 3-manifold in such a way that its fundamental group is a torsion-free subgroup of the Coxeter group, generated by reflections across the faces of a right-angled polyhedron [10]. Below we consider only compact polyhedra, which do not admit ideal vertices.

We start by recalling two nice recent results. Inoue 4 introduced two operations on right-angled polyhedra called decomposition and edge surgery, and proved that Löbell polyhedra (which will be a subject of discussion below) are universal in the following sense:

Theorem 1.1. 4, Theorem 9.1] Let $P_{0}$ be a right-angled hyperbolic polyhedron. Then there exists a sequence of disjoint unions of right-angled hyperbolic polyhedra $P_{1}, \ldots, P_{k}$ such that for $i=1, \ldots, k, P_{i}$ is obtained from $P_{i-1}$ by either a decomposition or an edge surgery, and $P_{k}$ is a set of Löbell polyhedra. Furthermore,

$$
\operatorname{vol}\left(P_{0}\right) \geqslant \operatorname{vol}\left(P_{1}\right) \geqslant \operatorname{vol}\left(P_{2}\right) \geqslant \ldots \geqslant \operatorname{vol}\left(P_{k}\right) \text {. }
$$

Atkinson [3] estimated the volume of a right-angled polyhedron in terms of the number of its vertices as follows:

Theorem 1.2. [3, Theorem 2.3] If $P$ is a compact right-angled hyperbolic polyhedron with $V$ vertices, then

$$
(V-2) \cdot \frac{v_{8}}{32} \leqslant \operatorname{vol}(P)<(V-10) \cdot \frac{5 v_{3}}{8},
$$

Date: July 17, 2018.

2010 Mathematics Subject Classification. 51M10; 51M25; 57M50.

Key words and phrases. Hyperbolic geometry, Coxeter polyhedra.

The first author was supported in part by the Slovenian Research Agency grants P1-02920101, J1-9643-0101 and J1-2057-0101. The second author was supported in part by the Russian Foundation for Basic Research grant 09-01-00255 and by the SO RAN - UrO RAN grant. 
where $v_{8}$ is the volume of a regular ideal octahedron, and $v_{3}$ is the volume of a regular ideal tetrahedron. There is a sequence of compact polyhedra $P_{i}$, with $V_{i}$ vertices such that $\operatorname{vol}\left(P_{i}\right) / V_{i}$ approaches $5 v_{3} / 8$ as $i$ goes to infinity.

A family of polyhedra $P_{i}$ suggested by Atkinson is described in the proof of [3, Prop. 6.4].

In this note we will demonstrate that Löbell polyhedra can serve as a suitable family realizing the upper bound. Thus these polyhedra play an important role not only in Theorem 1.1, but also in Theorem 1.2.

Let us denote by $\operatorname{vert}(R)$ the number of vertices of a right-angled polyhedron $R$. In this note we prove that $5 v_{3} / 8$ is a double limit point in the sense that it is the limit point of limit points for $\operatorname{ratios} \operatorname{vol}(R) / \operatorname{vert}(R)$.

Theorem 1.3. For any integer $k \geqslant 1$ there exists a series of compact right-angled polyhedra $R_{k}(n)$ in $\mathbb{H}^{3}$ such that

$$
\lim _{n \rightarrow \infty} \frac{\operatorname{vol}\left(R_{k}(n)\right)}{\operatorname{vert}\left(R_{k}(n)\right)}=\frac{k}{k+1} \cdot \frac{5 v_{3}}{8} .
$$

As one will see from the proof, $R_{1}(n)$ are Löbell polyhedra and $R_{k}(n)$ for $k>1$ are towers of them.

Moreover, in Corollary 4.3 we improve the lower estimate from Theorem 1.2 in the case $\operatorname{vert}(R) \leqslant 56$.

\section{LÖBELL POLYHEDRA AND MANIFOLDS.}

We introduced Löbell polyhedra in [10] as a generalization of a right-angled 14-hedron used in [5].

Recall that in order to give a positive answer to the question of the existence of "Clifford-Klein space forms" (that is, closed manifolds) of constant negative curvature, Löbell [5] constructed in 1931 the first example of a closed orientable hyperbolic 3-manifold. This manifold was obtained by gluing together eight copies of the right-angled 14-faced polytope (denoted below by $R(6)$ and shown in Fig. 1) with an upper and a lower basis both being regular hexagons, and a lateral surface given by 12 pentagons, arranged similarly as in the dodecahedron. Obviously, $R(6)$ can be considered as a generalization of a right-angled dodecahedron in the way of replacing basis pentagons to hexagons.

As shown in [10], the dodecahedron and $R(6)$ are part of a larger family of polyhedra. For each $n \geqslant 5$ we consider the right-angled polyhedron $R(n)$ in $\mathbb{H}^{3}$ with $(2 n+2)$ faces, two of which (viewed as the upper and lower bases) are regular $n$-gons, while the lateral surface is given by $2 n$ pentagons, arranged as one can easily imagine. Note that $R(5)$ is the right-angled dodecahedron (see Fig. 1). Existence of polyhedra $R(n)$ in $\mathbb{H}^{3}$ can be easy checked by involving Andreev's theorem [1].

An algebraic approach suggested in [10] admits a construction of both orientable and non-orientable closed hyperbolic 3-manifolds from eight copies of any bounded right-angled hyperbolic polyhedron. More exactly, any coloring of the faces of a right-angled polyhedron by four colors so that no two faces of the same color share an edge encodes a torsion-free subgroup of orientation preserving isometries which is a subgroup of the polyhedral Coxeter group of index eight. Thus, any four-coloring encodes an orientable hyperbolic 3-manifold obtained from eight 

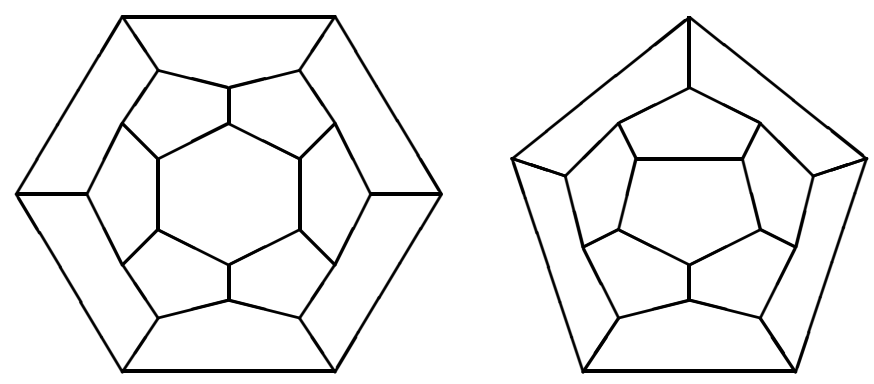

Figure 1. Polyhedra $R(6)$ and $R(5)$.

copies of a right-angled polyhedron. This approach also allows one to construct non-orientable hyperbolic 3-manifolds, but in this case five to seven colors are needed.

It was mentioned in [10] that the manifold constructed by Löbell can be encoded by some four-coloring of $R(6)$, and it was shown how to construct concrete orientable and non-orientable manifolds using eight copies of $R(n)$ for any $n \geqslant 5$. Closed orientable hyperbolic 3-manifolds encoded by four-colorings of $R(n), n \geqslant 5$, were called Löbell manifolds. (Observe that for each $n$ number of such manifolds do not need to be unique.) Polyhedra $R(n)$ can be naturally referred as Löbell polyhedra.

Various properties of Löbell manifolds were intensively studied: the volume formulae were obtained in [9] and [11], invariant trace fields for fundamental groups and their arithmeticity were numerically calculated in [2], many of Löbell manifolds were obtained in [8] as two-fold branched coverings of the 3-sphere, and two-sided bounds for complexity of Löbell manifolds were done in [7].

Since Lobachevsky's 1832 paper, the following Lobachevsky function has traditionally been used in volume formulae for hyperbolic polyhedra

$$
\Lambda(x)=-\int_{0}^{x} \log |2 \sin (t)| \mathrm{d} t .
$$

The volume formula for Löbell manifolds established in [11] implies the following formula for vol $R(n)$, since any Löbell manifolds indexed by $n$ is glued by isometries from eight copies of $R(n)$ :

Theorem 2.1. For all $n \geqslant 5$ we have

$$
\operatorname{vol}(R(n))=\frac{n}{2}\left(2 \Lambda\left(\theta_{n}\right)+\Lambda\left(\theta_{n}+\frac{\pi}{n}\right)+\Lambda\left(\theta_{n}-\frac{\pi}{n}\right)+\Lambda\left(\frac{\pi}{2}-2 \theta_{n}\right)\right),
$$

where

$$
\theta_{n}=\frac{\pi}{2}-\arccos \left(\frac{1}{2 \cos (\pi / n)}\right)
$$

It is easy to check that $\theta_{n} \rightarrow \pi / 6$ and $\frac{\operatorname{vol} R(n)}{n} \rightarrow \frac{5 v_{3}}{4}$ as $n \rightarrow \infty$. Here we use that $v_{3}=3 \Lambda(\pi / 3)=2 \Lambda(\pi / 6)$. Moreover, the asymptotic behavior of volumes of Löbell manifolds was established in [7, Prop. 2.10]. This implies trivially the description of the asymptotic behavior of $\operatorname{vol}(R(n))$ as $n$ tends to infinity. 
Proposition 2.1. The following inequalities hold for sufficiently large $n$ :

$$
\frac{5 v_{3}}{4} \cdot n-\frac{17 v_{3}}{2 n}<\operatorname{vol}(R(n))<\frac{5 v_{3}}{4} \cdot n .
$$

Since $\operatorname{vert}(R(n))=4 n$, we get

Corollary 2.1. The following inequalities hold for sufficiently large n:

$$
\frac{5 v_{3}}{16}-\frac{17 v_{3}}{8 n^{2}}<\frac{\operatorname{vol}(R(n))}{\operatorname{vert}(R(n))}<\frac{5 v_{3}}{16} .
$$

\section{Proof of Theorem 1.3.}

We will use Löbell polyhedra $R(n)$ as building blocks to construct right-angled polyhedra with necessary properties. Let us present polyhedra $R(n)$ by their lateral surfaces as it is done in Fig. 2 for polyhedra $R(6)$ and $R(5)$, keeping in mind that left and right sides are glued together.
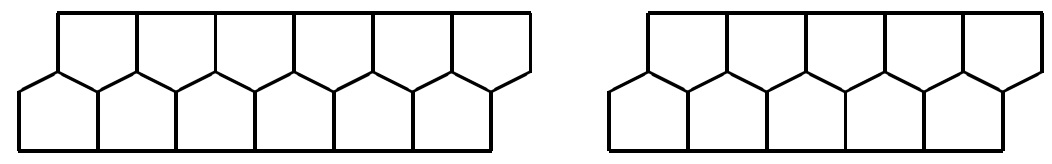

Figure 2. Polyhedra $R(6)$ and $R(5)$.

For integer $k \geq 1$ denote by $R_{k}(n)$ the polyhedron constructed from $k$ copies of $R(n)$ gluing them along $n$-gonal faces similar to a tower. In particular, $R_{1}(n)=$ $R(n)$. The polyhedron $R_{3}(6)$ is presented in Fig. 3 .

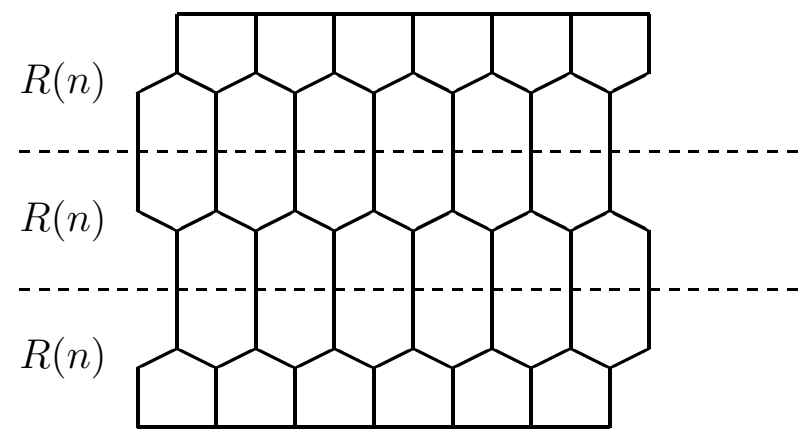

Figure 3. Polyhedron $R_{3}(6)$.

Obviously, $R_{k}(n)$ is a right-angled polyhedron with $n$-gonal top and bottom and the lateral surface formed by $2 n$ pentagons and $(k-1) n$ hexagons.

Since $\operatorname{vol}\left(R_{k}(n)\right)=k \cdot \operatorname{vol}(R(n))$, Proposition 2.1 implies that for sufficiently large $n$

$$
k \cdot \frac{5 v_{3}}{4} \cdot n-k \cdot \frac{17 v_{3}}{2 n}<\operatorname{vol}\left(R_{k}(n)\right)<k \cdot \frac{5 v_{3}}{4} \cdot n .
$$

Since vert $R_{k}(n)=(2 k+2) n$, we obtain

$$
\frac{k}{k+1} \cdot \frac{5 v_{3}}{8}-\frac{k}{k+1} \cdot \frac{17 v_{3}}{4 n^{2}}<\frac{\operatorname{vol}\left(R_{k}(n)\right)}{\operatorname{vert}\left(R_{k}(n)\right)}<\frac{k}{k+1} \cdot \frac{5 v_{3}}{8} .
$$


Thus family of right-angled polyhedra $R_{k}(n)$ is such that for any integer $k \geqslant 1$

$$
\lim _{n \rightarrow \infty} \frac{\operatorname{vol}\left(R_{k}(n)\right)}{\operatorname{vert}\left(R_{k}(n)\right)}=\frac{k}{k+1} \cdot \frac{5 v_{3}}{8}
$$

and the upper bound $5 v_{3} / 8$ is a double limit point in the sense that it is the limit of above limit points as $k \rightarrow \infty$ :

$$
\lim _{k, n \rightarrow \infty} \frac{\operatorname{vol}\left(R_{k}(n)\right)}{\operatorname{vert}\left(R_{k}(n)\right)}=\frac{5 v_{3}}{8} .
$$

Thus, the theorem is proved.

\section{Other volume estimates.}

Since 1-skeleton of a right-angled compact hyperbolic polyhedron $P$ is a trivalent plane graph, one can easy see that Euler formula for a polyhedron implies

$$
V=2 F-4,
$$

where $V$ is number of vertices of $P$ and $F$ is number of its faces. Moreover, Euler formula implies also that $P$ has at least 12 faces (this smallest number of faces corresponds to a dodecahedron). Thus, Theorem 1.2 implies the following result.

Corollary 4.1. If $P$ is a compact right-angled hyperbolic polyhedron with $F$ faces, then

$$
(F-3) \cdot \frac{v_{8}}{16} \leqslant \operatorname{vol}(P)<(F-7) \cdot \frac{5 v_{3}}{4} .
$$

We recall that constants $v_{3}$ and $v_{8}$ are

$$
v_{3}=3 \Lambda(\pi / 3)=1.0149416064096535 \ldots
$$

and

$$
v_{8}=8 \Lambda(\pi / 4)=3.663862376708876 \ldots
$$

Since a right-angled hyperbolic $n$-gon has area $\pi / 2 \cdot(n-4)$, the lateral surface area of a compact hyperbolic right-angled polyhedron $P$ with $F$ faces is equal to $\pi \cdot(F-6)$. Thus, Corollary 4.1 implies the following result.

Corollary 4.2. If $P$ is a compact right-angled hyperbolic polyhedron with lateral surface area $S$, then

$$
(S / \pi+3) \cdot \frac{v_{8}}{16} \leqslant \operatorname{vol}(P)<(S / \pi-1) \cdot \frac{5 v_{3}}{4} .
$$

Observe, that Theorem 2.1 can be used to show that the volume function $\operatorname{vol} R(n)$ is a monotonic increasing function of $n$ (see [4] and [7] for proofs), and to calculate volumes of Löbell polyhedra. In particular,

$$
\operatorname{vol} R(5)=4.306 \ldots, \quad \operatorname{vol} R(6)=6.023 \ldots, \quad \operatorname{vol} R(7)=7.563 \ldots
$$

Together with Theorem 1.1 it gives that the right-angled hyperbolic polyhedron of smallest volume is $R(5)$ (a dodecahedron) and the second smallest is $R(6)$. Thus, if a compact right-angled hyperbolic polyhedron $P$ is differ of a dodecahedron, then

$$
\operatorname{vol}(P) \geqslant 6.023 \ldots
$$

Thus, we get the following 
Corollary 4.3. If $P$ is a compact right-angled hyperbolic polyhedron different than a dodecahedron, having $V$ vertices and $F$ faces. Then

and

$$
\operatorname{vol}(P) \geqslant \max \left\{(V-2) \cdot \frac{v_{8}}{32}, 6.023 \ldots\right\}
$$

$$
\operatorname{vol}(P) \geqslant \max \left\{(F-3) \cdot \frac{v_{8}}{16}, 6.023 \ldots\right\} .
$$

The estimates from Corollary 4.3 improve the lower estimate from Theorem 1.2 for $V \leqslant 54$ and the lower estimate from Corollary 4.1 for $F \leqslant 29$.

\section{REFERENCES}

[1] E. M. Andreev, On convex polyhedra in Lobachevsky space, Math. USSR Sbornik, 10(3) (1970), 413-440.

[2] O. Antolin-Camarena, G. Maloney, R.K.W. Roeder, Computing arithmetic invariants for hyperbolic reflection groups, In Complex Dynamics: Families and Friends (D. Schleicher editor) pages 571-605, A.K. Peters, 2008.

[3] C. K. Atkinson, Volume estimates for equiangular hyperbolic Coxeter polyhedra, Algebraic \& Geometric Topology, 9 (2009), 1225-1254.

[4] T. Inoue, Organizing volumes of right-angled hyperbolic polyhedra, Algebraic \& Geometric Topology, 8 (2008), 1523-1565.

[5] F. Löbell, Beispiele geschlossene dreidimensionaler Clifford - Kleinischer Räume negative Krümmung, Ber. Verh. Sächs. Akad. Lpz., Math.-Phys. Kl. 83 (1931), 168-174.

[6] S. Matveev, C. Petronio, A. Vesnin, Two-sided complexity bounds for Löbell manifolds, Doklady Mathematics, 76(2) (2007), 689-691.

[7] S. Matveev, C. Petronio, A. Vesnin, Two-sided asymptotic bounds for the complexity of some closed hyperbolic three-manifolds, Journal of the Australian Math. Soc. 86(2) (2009), 205-219.

[8] A. Mednykh, A. Vesnin, Three-dimensional hyperelliptic manifolds and hamiltonian graphs, Siberian Math. J. 40(4) (1999), 628-643.

[9] A. Mednykh, A. Vesnin, Löbell manifolds revised, Siberian Electronic Mathematical Reports, 4 (2007), 605-609.

[10] A. Vesnin, Three-dimensional hyperbolic manifolds of Löbell type, Siberian Math. J. 28(5) (1987), 731-734.

[11] A. Vesnin, Volumes of three-dimensional hyperbolic Löbell manifolds, Math. Notes 64(1) (1998), 15-19.

Faculty of Mathematics and Physics, and Faculty of Education, University of Luubluana, P.O.Box 2964, SI-1001 Luubluana, Slovenia

E-mail address: dusan.repovs@guest.arnes.si

Sobolev Institute of Mathematics, Pr. ak. Koptyuga 4, Novosibirsk, 630090, Russia and Department of Mathematics, Omsk State Technical University, PR. Mira 11, OMsk, 644050, Russia

E-mail address: vesnin@math.nsc.ru 